\title{
Cysteamine Enhances the Procoagulant Activity of Factor VIII-East Hartford, A Dysfunctional Protein due to a Light Chain Thrombin Cleavage Site Mutation (Arginine-1689 to Cysteine)
}

\author{
Ashraf M. Aly, Morio Arai, and Leon W. Hoyer \\ Holland Laboratory, American Red Cross Blood Services, Rockville, Maryland 20855
}

\begin{abstract}
We have recently identified the molecular defect responsible for cross-reacting material-positive hemophilia $A$ in two unrelated patients in which the substitution of cysteine for arginine1689 (Factor VIII-East Hartford[FVIII-EH]) abolishes a critical Factor VIII light chain thrombin cleavage site. As other mutant proteins with a cysteine for arginine substitution have been modified in the presence of cysteamine, we have determined the effect of this and other reducing agents on FVIII-EH function. Cysteamine concentrations between 0.1 and $10 \mathrm{mM}$ caused dose- and time-dependent increases in FVIII-EH VIII:C activity, as much as 14-fold (to 35 and $62 \mathrm{U} / \mathrm{dl}$ for the two patients tested). Comparable data were obtained in a standard one-stage VIII:C coagulation assay and in a chromogenic substrate assay measuring Factor Xa generation. Thrombin cleavage of the FVIII-EH light chain in the presence of cysteamine was documented by immunoadsorption and analysis. Cystamine and cysteamine-S-phosphate, similar compounds that do not possess a free thiol group, had no effect. Cysteamine augmentation of FVIII-EH VIII:C was abolished by the simultaneous addition of $\mathrm{N}$-ethyl maleimide or iodoacetamide, but these sulfhydryl blocking agents did not prevent the VIII:C increase and light chain cleavage by thrombin if the plasma samples were dialyzed to remove the inhibitors before adding the cysteamine. However, incubation with DTT before iodoacetamide prevented the cysteamine effect after dialysis. These data suggest that when isolated from patient plasma, FVIIIEH cysteine-1689 is present in a disulfide bond. This bond is cleaved by cysteamine to form a new mixed disulfide, a pseudolysine that restores a thrombin cleavage site that is essential for procoagulant function.(J. Clin. Invest. 1992. 89:1375-1381.) Key words: hemophilia $\bullet$ cross-reacting material $\bullet$ penicillamine - disulfide • sulfhydryl
\end{abstract}

This work was presented in part at the 1989 annual meeting of the American Society of Hematology, Atlanta, GA, and at the 1990 annual meeting of the American Society of Clinical Investigation, Washington, DC. A. M. Aly performed this work in partial fulfillment of the Ph.D. requirements in the Graduate Genetics Program at George Washington University.

Address reprint requests to L. W. Hoyer, M.D., Holland Laboratory, American Red Cross Blood Services, 15601 Crabbs Branch Way, Rockville, MD 20855.

Received for publication 19 August 1991 and in revised form 30 December 1991.

J. Clin. Invest.

(c) The American Society for Clinical Investigation, Inc.

0021-9738/92/05/1375/07 \$2.00

Volume 89, May 1992, 1375-1381

\section{Introduction}

Factor VIII (antihemophilic factor) participates in blood coagulation as an essential cofactor in the activation of Factor $\mathrm{X}$ by Factor IXa in the presence of phospholipid and calcium. It is modified by several proteolytic cleavages in a process that leads to activation and subsequent inactivation of the molecule (13). The mature single-chain protein of 2,332 amino acids is proteolytically processed to generate amino-terminal heavy chain polypeptides ranging in size from 92 to $200 \mathrm{kD}$ and an $80-\mathrm{kD}$ carboxy-terminal light chain. Factor VIII procoagulant activity is then generated by thrombin cleavages at arginine740 and arginine-372 to yield 54- and 44-kD heavy chain fragments and at arginine- 1689 to yield the $72-\mathrm{kD}$ light chain fragment. The heavy chain and light chain cleavages are critical for Factor VIII activation (4).

Hemophilia A, an X-linked inherited bleeding disorder, is caused by reduction or absence of Factor VIII activity. Nonfunctional, immunoreactive Factor VIII-like protein, at levels comparable to those in normal plasma, has been detected in $10 \%$ of plasmas from patients with mild or moderate hemophilia A (5). Such plasmas are termed cross-reacting material positive (CRM-positive). ${ }^{1}$ We have recently identified the molecular defect that is responsible for CRM-positive hemophilia $A$ in two unrelated patients. In the dysfunctional protein designated Factor VIII-East Hartford (FVIII-EH), substitution of cysteine for arginine-1689 abolishes the critical Factor VIII light chain thrombin cleavage site (6).

The possibility that FVIII-EH might be susceptible to chemical modification is suggested by data for apo E-2, a mutant protein in which a cysteine for arginine substitution blocks lipoprotein interaction with receptors. Type III hyperlipoproteinemia is a pathological lipid disorder associated with the apo E-2 homozygous state. Weisgraber et al. (7) have demonstrated that cysteamine (beta-mercaptoethylamine) treatment of apo E-2 in vitro converts the mutant cysteine to a positively charged lysine analogue and markedly increases the binding of apo E-2 to fibroblast lipoprotein receptors. Gahl et al. (8) showed that doses of cysteamine used in the treatment of the lysosomal storage disorder, cystinosis, modify apo E-2 in vivo in a way that can be detected by changes in the isoelectric focusing pattern. In cystinosis itself, the beneficial effect of cysteamine is thought to be due to disulfide interchange that generates products that can traverse the lysosomal membrane, overcoming the critical defect in cystine transport (9).

The data for apoprotein E-2 suggested that cysteamine

1. Abbreviations used in this paper: BBS, barbital-buffered saline; CRM, cross-reacting material; FVIII-EH, Factor VIII-East Hartford; IA, iodoacetamide; NEM, $N$-ethyl maleimide; VIII:Ag, Factor VIII antigen; VIII:C, Factor VIII procoagulant activity. 
might have an effect on FVIII-EH. Indeed, if this chemical could alter the properties of the mutant protein, it might be an alternative to replacement therapy with Factor VIII concentrates prepared from pooled human plasma. As a first step toward the evaluation of cysteamine as a therapy in patients with FVIII-EH, we have determined the in vitro effects of cysteamine on FVIII-EH function.

\section{Methods}

Materials. Cysteamine $\mathrm{HCl}$ (2-mercaptoethylamine), cysteamine-Sphosphate, D-L-cysteine, glutathione, D-L-penicillamine, cystamine, DTT, 2-bromethylamine $\mathrm{HCl}$, iodoacetamide (IA), and $\mathrm{N}$-ethylmaleimide (NEM) were purchased from Sigma Chemical Co. (St. Louis, MO). 2-Dimethylaminoethanethiol $\mathrm{HCl}$ was purchased from Aldrich Chemical Co. (Milwaukee, WI). All chemicals were kept desiccated at $4^{\circ} \mathrm{C}$ until they were dissolved in $150 \mathrm{mM} \mathrm{NaCl} / 20 \mathrm{mM}$ Tris- $\mathrm{HCl}, \mathrm{pH}$ 7.4. These solutions were then held at $4^{\circ} \mathrm{C}$. Chromogenic assay of Factor VIII was carried out with reagents purchased from American Bioproducts (Parsippany, $\mathrm{NJ}$ ).

The characteristics of the CRM-positive hemophilia A plasmas and clinical data for patients ARC-5 and ARC-10 have been described in previous reports $(6,10)$.

Factor VIII measurements. Factor VIII procoagulant activity (VIII:C) was measured in a one-stage assay using Factor VIII-deficient plasma as substrate (11). VIII:C was also determined using a chromogenic assay in which Factor Xa production was measured (12). Factor VIII antigen (VIII:Ag) was measured by immunoradiometric assay using ${ }^{125}$ I-labeled $\mathrm{Fab}^{\prime}$ prepared from the plasma of a patient with an alloantibody to Factor VIII (13). vWf antigen (vWf:Ag) was measured by immunoradiometric assay using a rabbit antibody (14). Citrate-anticoagulated normal plasma, pooled from 15 donors, served as a standard (1 U/ml) for VIII:C, VIII:Ag, and vWf:Ag measurements and was used as the control for immunopurification studies.

Immunopurification of Factor VIII. The procedure for immunopurifying Factor VIII from small volumes of plasma has been published (10). Patient plasmas or pooled normal plasma were incubated with immunoadsorbent beads prepared by coupling IgG from a high titer Factor VIII inhibitor plasma to agarose. The adsorbed Factor VIII protein was incubated in some cases with highly purified human thrombin (0.2-5 U thrombin/U VIII:Ag). As previously described, untreated or thrombin-treated Factor VIII was then eluted with SDS and identified by immunoblotting using monoclonal anti-Factor VIII antibodies after SDS-PAGE (10).

Determination of VIII:C activity in plasmas incubated with reducing agents. CRM-positive hemophilic plasmas (ARC-5 and ARC-10) and normal pooled plasma were incubated at $37^{\circ} \mathrm{C}$ with equal volumes of dilutions of cysteamine $\mathrm{HCl}$ or other reducing agents or chemicals in barbital-buffered saline (BBS) (13) or with BBS. VIII:C assays for these mixtures were then obtained using normal plasma incubated with BBS as the standard.

Evaluation of thrombin-cleavage at arginine-1689 in samples incubated with cysteamine. Mixtures of immunoadsorbent beads with normal or FVIII-EH plasmas were incubated with gentle agitation at room temperature for $16 \mathrm{~h}$ to ensure complete adsorption. They were then incubated with cysteamine ( $10 \mathrm{mM}$ final concentration) for $30 \mathrm{~min}$ at $37^{\circ} \mathrm{C}$. The beads were then washed and processed as noted above (10).

\section{Results}

Effect of cysteamine on Factor VIII activity. The addition of cysteamine to normal plasma for $1 \mathrm{~h}$ at $37^{\circ} \mathrm{C}$ had little effect on one-stage VIII:C coagulation assays at concentrations $<1 \mathrm{mM}$. Higher concentrations caused VIII:C loss (Fig. 1). For a fixed cysteamine concentration, the inhibitory effect on normal plasma VIII:C was also time dependent (Fig. 2). By coagulation

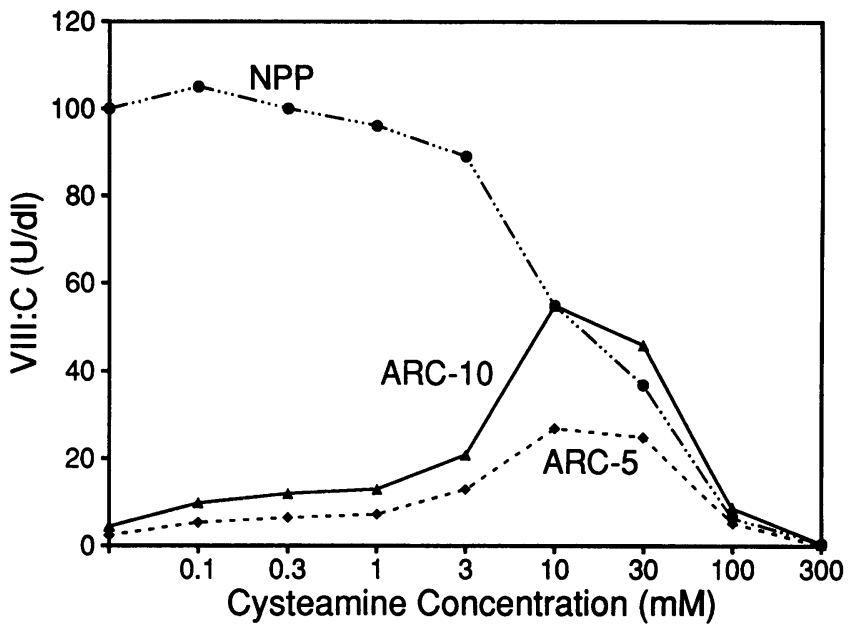

Figure 1. Effect of cysteamine on FVIII-EH VIII:C activity. Mixtures of FVIII-EH ( $A R C$-5 and $A R C-10)$ or pooled normal plasma (NPP) with cysteamine (final concentration indicated) were incubated at $37^{\circ} \mathrm{C}$ for $60 \mathrm{~min}$ before VIII:C measurement by a one-stage coagulation assay.

assay, addition of cysteamine never increased normal plasma VIII:C. The inhibitory effect of high concentrations of cysteamine is not specific for Factor VIII, as these concentrations had a similar inhibitory effect on the apparent Factor IX procoagulant activity of normal plasma (data not shown).

In contrast, addition of cysteamine to the two CRM-positive FVIII-EH hemophilic plasmas caused a consistent concentration- and time-dependent increase in VIII:C activity (Figs. 1 and 2). The maximum increase for ARC-5 was 14-fold (from 2.5 to $35 \mathrm{U} / \mathrm{dl}$ ), and for ARC-10 it was 13.8-fold (from 4.5 to 62 $\mathrm{U} / \mathrm{dl}$ ). At concentrations $>10 \mathrm{mM}$, or for incubation at 10 $\mathrm{mM}$ for $>30 \mathrm{~min}$, cysteamine had a similar inhibitory effect on the VIII:C activity of the hemophilic plasmas and normal plasma. The concentration- and time-dependent cysteamine effect was observed in two separate studies.

When similar studies were carried out with CRM-negative hemophilia A plasmas (three different samples), CRM-reduced plasmas (three samples), and a CRM-positive hemophilic plasma in which the molecular defect is substitution of histidine for arginine-372 (ARC-1 in reference 11), there was no increase in VIII: $C$ when $10 \mathrm{mM}$ cysteamine was incubated with the plasma sample for $1 \mathrm{~h}$ at $37^{\circ} \mathrm{C}$.

The VIII:C detected after cysteamine treatment of ARC-5 plasma was authentic Factor VIII cofactor activity, not a result of the modification of some other protein, since it was inactivated by a Factor VIII inhibitor (Ab9) (15) to the same extent as was normal plasma VIII:C.

Generally similar cysteamine effects were noted when VIII:C was measured by chromogenic assay instead of a onestage coagulation assay (Fig. 3). Unexpectedly, cysteamine concentrations $>3 \mathrm{mM}$ increased the VIII:C assay values of normal plasma, to an apparent maximum of $155 \mathrm{U} / \mathrm{dl}$. The reason for the more rapid Factor $\mathrm{X}$ cleavage in the presence of cysteamine is not known. For this reason, data for FVIII-EH plasma incubated with cysteamine are best expressed as a percentage of the VIII:C for normal plasma incubated with the same cysteamine concentration. ARC-10 VIII:C increased from $<1 \%$ in the absence of cysteamine to $>20 \%$ at cysteamine concentrations of $3 \mathrm{mM}$ or greater. Similar studies 


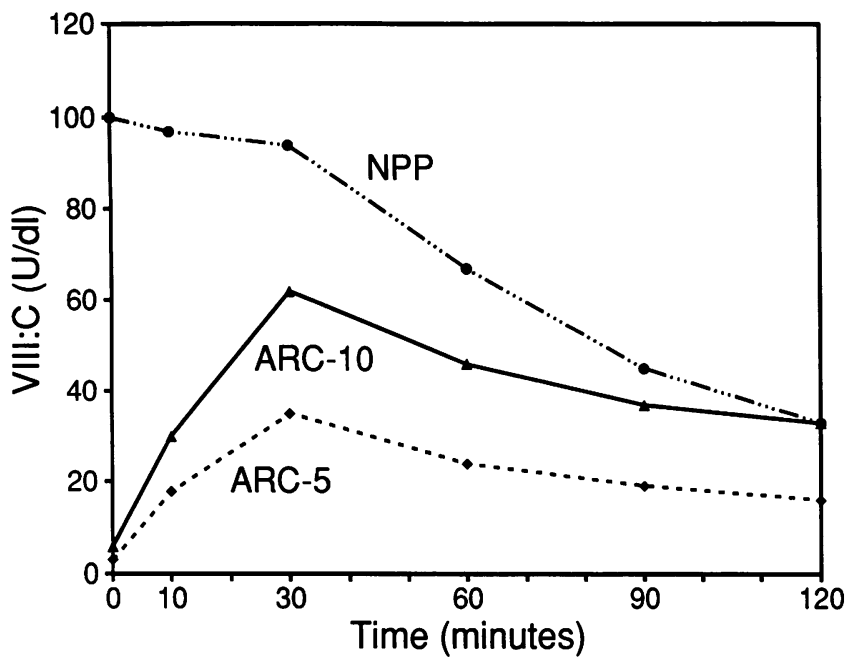

Figure 2. Time course of the cysteamine effect on VIII:C activity. Mixtures of plasma and $10 \mathrm{mM}$ cysteamine (final concentration) were incubated at $37^{\circ} \mathrm{C}$ before VIII:C measurement by a one-stage coagulation assay.

carried out with a CRM-negative hemophilia A plasma detected no VIII:C by chromogenic assay when $10 \mathrm{mM}$ cysteamine was incubated with the plasma for $1 \mathrm{~h}$ at $37^{\circ} \mathrm{C}$.

Effect of cysteamine on thrombin cleavage of FVIII-EH light chains. To determine the mechanism of the cysteamine effect, thrombin was added to immunoadsorbed FVIII-EH that had been incubated with cysteamine (10). A mixture of FVIIIEH plasma and immunoadsorbent beads was held at room temperature for $16 \mathrm{~h}$, cysteamine $(10 \mathrm{mM})$ was added for an additional 30-min incubation, the beads were washed, and thrombin was added for $1 \mathrm{~h}$. Under these conditions, there was complete cleavage of the ARC-5 Factor VIII light chain (Fig. 4, lane 7), whereas there was no light chain cleavage of FVIII-EH

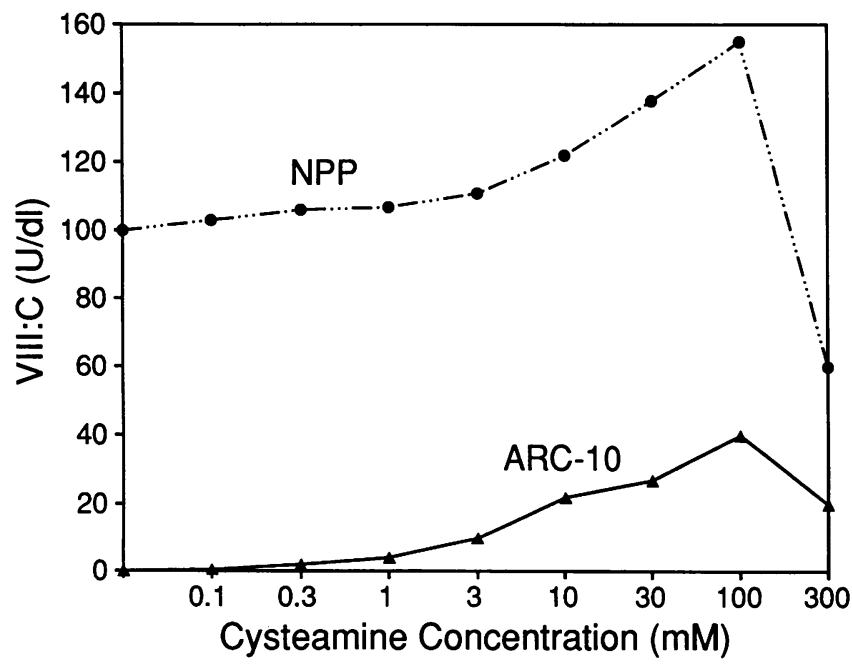

Figure 3. Effect of cysteamine on VIII:C measurement by chromogenic substrate assay. Mixtures of ARC-10 plasma or pooled normal plasma with cysteamine (final concentration indicated) were incubated at $37^{\circ} \mathrm{C}$ for $30 \mathrm{~min}$ before VIII:C measurement by a chromogenic substrate assay. The standard used for this assay was a mixture of equal volumes of normal plasma and $\mathrm{BBS}$ held at $37^{\circ} \mathrm{C}$ for $30 \mathrm{~min}$.

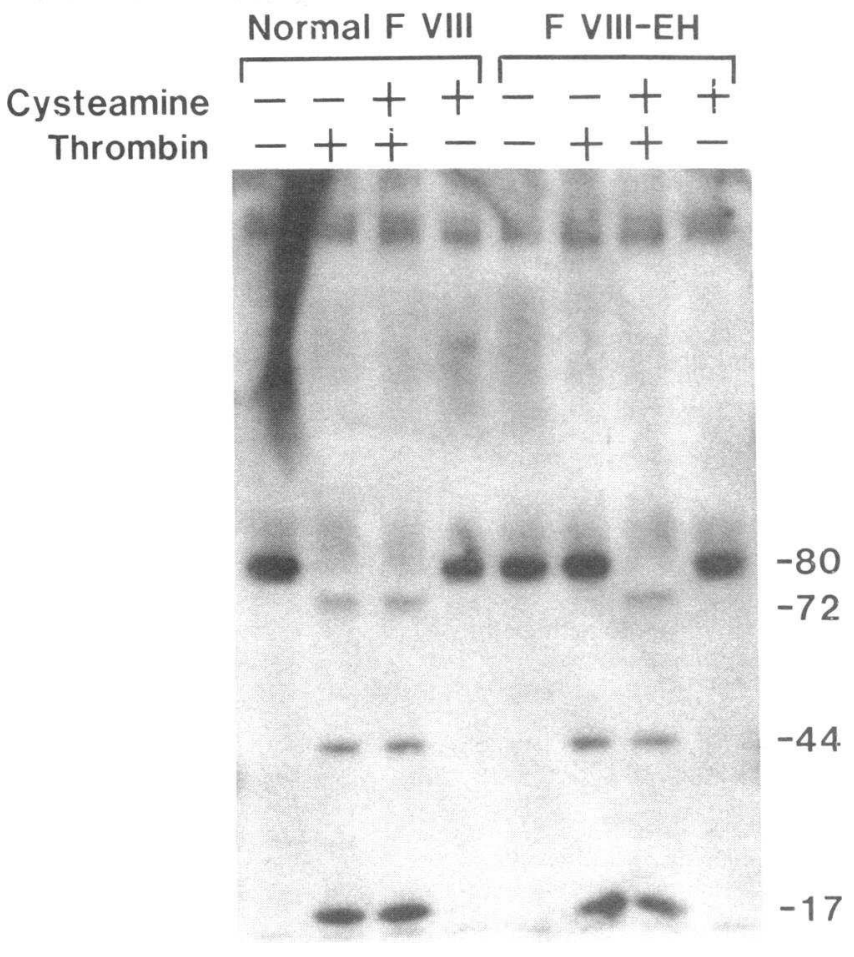

\section{Lane $\begin{array}{lllllllll}1 & 2 & 3 & 4 & 5 & 6 & 7 & 8\end{array}$}

Figure 4. Immunoblots showing the effect of cysteamine on FVIII-EH cleavage by thrombin. Immunoadsorbed plasma Factor VIII (2 U VIII:Ag) was incubated with cysteamine ( $10 \mathrm{mM}$ final concentration) or BBS for $30 \mathrm{~min}$ at $37^{\circ} \mathrm{C}$, the immunoadsorbent beads were washed, and they were then treated with thrombin ( $10 \mathrm{U}$ in $0.1 \mathrm{ml})$ or BBS for $60 \mathrm{~min}$ at room temperature before SDS elution. Analysis of the proteins after SDS-PAGE and transfer to nitrocellulose was done with a mixture of three monoclonal anti-FVIII antibodies (10). In the study illustrated here, ARC-5 was the FVIII-EH plasma analyzed. Comparable data were obtained with ARC-10.

that had not been incubated with cysteamine (lane 6). As expected, there was complete thrombin cleavage of normal Factor VIII heavy and light chains as well as the ARC-5 heavy chain (Fig. 4, lanes 2, 3, 6, and 7). Only partial cleavage of the FVIII-EH light chain was observed after incubation with cysteamine when lower thrombin concentrations were used (e.g., $2 \mathrm{U}$ thrombin/ $0.2 \mathrm{ml}$ final volume for $2 \mathrm{U}$ VIII:Ag adsorbed to the beads, in contrast to $10 \mathrm{U}$ thrombin/0.1 ml in the Fig. 4 experiment). For normal Factor VIII light chains, complete cleavage was obtained with thrombin concentrations as low as $0.4 \mathrm{U}$ thrombin/0.2 $\mathrm{ml}(10)$.

The conditions necessary for the cysteamine effect were quite specific, since FVIII-EH light chain cleavage was not detected if the immunoadsorbent beads were separated from FVIII-EH plasma before cysteamine was added. That the cysteamine effect on adsorbed FVIII-EH required the simultaneous presence of another plasma component(s) was established by determining the extent of light chain cleavage when washed FVIII-EH bound to immunoadsorbent beads was suspended in CRM-negative hemophilia A or severe von Willebrand's disease plasma before cysteamine addition. In these experiments, the extent of thrombin cleavage was as great as when cysteamine was added to immunoadsorbent beads that were suspended in FVIII-EH plasma (data not shown). The 


\begin{tabular}{|c|c|c|c|}
\hline $\begin{array}{l}\text { Material } \\
\text { tested }\end{array}$ & Structure & $\begin{array}{l}\text { VIII:C increase } \\
\text { after incubation }\end{array}$ & $\begin{array}{c}\text { Optimal } \\
\text { concentration }\end{array}$ \\
\hline & & & $m M$ \\
\hline Cysteamine & $\mathrm{HS}-\mathrm{CH}_{2}-\mathrm{CH}_{2}-\mathrm{NH}_{2}$ & 10-14-fold & 10 \\
\hline Penicillamine & $\begin{array}{c}\stackrel{\mathrm{CH}}{\mathrm{CH}}-\mathrm{NH}_{2} \\
\stackrel{\mathrm{C}}{\mathrm{C}}-\mathrm{CH}-\mathrm{COOH} \\
\stackrel{\mathrm{CH}}{3}\end{array}$ & 5-6-fold & $80-150$ \\
\hline DTT & $\begin{array}{c}\mathrm{HS}-\mathrm{CH}_{2}-\mathrm{CH}-\mathrm{CH}-\mathrm{CH}_{2}-\mathrm{SH} \\
\mathrm{CH}_{3}\end{array}$ & 5-6-fold & 1 \\
\hline Dimethylaminoethanethiol & $\mathrm{HS}-\mathrm{CH}_{2}-\mathrm{CH}_{2}-\mathrm{N}$ & 3-fold & 30 \\
\hline Cystamine & $\begin{array}{l}\mathrm{S}-\mathrm{CH}_{2}-\mathrm{CH}_{2}-\mathrm{NH}_{2} \\
\text { S- } \mathrm{CH}_{2}-\mathrm{CH}_{2}-\mathrm{NH}_{2}\end{array}$ & None & \\
\hline Cysteamine-S-phosphate & $\mathrm{PO}_{4}-\mathrm{S}-\mathrm{CH}_{2}-\mathrm{CH}_{2}-\mathrm{NH}_{2}$ & None & \\
\hline Cysteine & 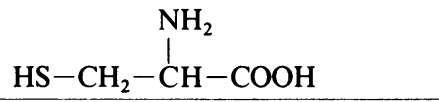 & None & \\
\hline
\end{tabular}

Equal volumes of each compound diluted in barbital-buffered saline (BBS) and FVIII-EH plasma were incubated at $37^{\circ} \mathrm{C}$ for $30 \mathrm{~min}$ before one-stage Factor VIII coagulation assay. The VIII:C increase is the ratio of VIII:C values for FVIII-EH incubated with the compound and FVIII-EH incubated with BBS alone. FVIII-EH, factor VIII-East Hartford; VIII:C, factor VIII procoagulant activity.

nature of this component(s) has not yet been further characterized, but the requirement may be related to the specific conditions under which cysteamine was added to immunoadsorbed Factor VIII. Cysteamine added to highly purified FVIII-EH/ vWF complexes generated VIII:C activity to an extent comparable to that in whole ARC-10 plasma, indicating that there is no requirement for an additional plasma component when the cysteamine effect is evaluated in solution (15a).

Effect of other reducing agents. To better understand the mechanism of cysteamine action, we determined the effects of other reducing agents on FVIII-EH procoagulant activity. In each case, equal volumes of plasma and test compound were incubated for $30 \mathrm{~min}$ at $37^{\circ} \mathrm{C}$ before VIII:C assay (Table I). The essential role of the free sulfhydryl group was established by demonstrating that neither cystamine nor cysteamine- $S$-phosphate had any effect on FVIII-EH VIII:C activity. Two compounds with structures quite distinct from that of cysteamine, penicillamine and DTT, also produced consistent, dose-dependent, five- to sixfold VIII:C increases (Table I). The optimal concentrations were $1 \mathrm{mM}$ for DTT and $80 \mathrm{mM}$ for penicillamine. A smaller increase was observed with dimethylaminoethanethiol, whereas cysteine and the tripeptide, glutathione, had no effect. An alternative reagent, bromoethylamine, had no effect on FVIII-EH plasma VIII:C at concentrations between 0.1 and $100 \mathrm{mM}$.

Effect of thiol inhibitors. The effects of NEM and IA, potent thiol blocking agents, were then determined. When equal volumes of plasma and either NEM or IA were incubated for 30 min at $37^{\circ} \mathrm{C}$, both compounds reduced normal plasma VIII:C levels in a dose-dependent manner at concentrations $>0.1$ (NEM) or $10 \mathrm{mM}$ (IA). However, the simultaneous addition of
$20 \mathrm{mM}$ IA and $10 \mathrm{mM}$ cysteamine to ARC-5 plasma completely blocked the cysteamine-induced VIII:C increase in FVIII-EH (Table II, row 3). Comparable results were obtained when $20 \mathrm{mM}$ NEM and $10 \mathrm{mM}$ cysteamine were simultaneously added to ARC-5 plasma (data not shown).

The inhibition of the cysteamine effect by the simultaneous addition of IA or NEM could be due to an interaction with the mutant cysteine-1689 sulfhydryl group or it could be due to the formation of a covalent bond with the cysteamine sulfhydryl group. This distinction was addressed in a series of experiments in which the thiol inhibitors were incubated with FVIII-EH plasma and then dialyzed before cysteamine treatment. Although there was significant VIII:C loss during the processing steps, a consistent pattern was seen in four separate studies using both ARC-5 and ARC-10 treated with IA or NEM. A typical experiment using IA is summarized in rows 5-7 of Table II. Incubation of ARC-10 plasma with IA before dialysis did not prevent an VIII:C rise when cysteamine was subsequently added (row 5). Compared with the VIII:C level in normal plasma treated in the same way, ARC-10 VIII:C was similar to that when cysteamine was added in the absence of a thiol inhibitor (row 2). Prior incubation of ARC-10 with DTT changed this pattern, however. Even though DTT alone had a modest VIII:C-augmenting effect (row 6), addition of DTT before IA (and subsequent dialysis to remove both compounds) reduced the subsequent cysteamine-induced VIII:C increase (row 7). These data suggest that IA does not react with FVIII-EH cysteine-1689 until a disulfide bond is reduced by DTT. Then IA or NEM can form a covalent bond with cysteine-1689 that prevents its subsequent interaction with cysteamine to generate a thrombin cleavage site. 
Table II. Effect of Iodoacetamide on the Cysteamine Effect

\begin{tabular}{|c|c|c|c|c|c|c|c|}
\hline \multirow[b]{2}{*}{$\begin{array}{l}\text { Sample } \\
\text { No. }\end{array}$} & \multirow[b]{2}{*}{$\begin{array}{c}\text { DTT } \\
5 \mathrm{mM}\end{array}$} & \multirow[b]{2}{*}{$\begin{array}{l}\text { Iodoacetamide } \\
20 \mathrm{mM}\end{array}$} & \multirow[b]{2}{*}{ Dialysis } & \multirow[b]{2}{*}{$\begin{array}{l}\text { Cysteamine } \\
10 \mathrm{mM}\end{array}$} & \multicolumn{2}{|c|}{ VIII:C } & \multirow[b]{2}{*}{ ARC-10 } \\
\hline & & & & & Normal & ARC-10 & \\
\hline & & & & & \multicolumn{2}{|c|}{$U / d l$} & $\%$ of normal \\
\hline 1 & - & - & - & - & 100 & 2 & 2 \\
\hline 2 & - & - & - & Cysteamine & 61 & 16 & 26 \\
\hline 3 & - & IA & - & Cysteamine & 95 & 2 & 2 \\
\hline 4 & - & IA & - & - & 80 & $<1$ & $<1$ \\
\hline 5 & - & IA & + & Cysteamine & 25 & 8 & 32 \\
\hline 6 & DTT & - & + & - & 34 & 7 & 21 \\
\hline 7 & DTT & IA & + & Cysteamine & 22 & 3 & 14 \\
\hline
\end{tabular}

Plasma samples were sequentially incubated with DTT and/or iodoacetamide (IA) and/or cysteamine (or BBS where indicated by -) for 30 min at $37^{\circ} \mathrm{C}$. Samples 5-7 were dialyzed against BBS before the final addition of cysteamine or BBS. One-stage coagulation assay using pooled normal plasma used as the assay standard. The relative ARC-10 level, compared with normal plasma incubated and/or dialyzed in the same way, is indicated in a separate column.

This hypothesis was tested by examining the effects of these additions on FVIII-EH light chain cleavage. Normal or ARC-5 plasmas were briefly incubated with DTT before IA was added, the samples were dialyzed, and the treated plasmas were incubated with anti-FVIII immunosorbent beads. The plasma/ bead suspensions were then incubated with cysteamine, the beads were washed, and the adsorbed Factor VIII was treated with thrombin. Whereas IA alone did not block cysteamine-dependent thrombin cleavage of the FVIII-EH light chain (Fig. 5, lane 4), IA added after incubation with DTT completely blocked cysteamine-dependent light chain cleavage (lane 1 ).

\section{Discussion}

Cysteamine (beta-mercaptoethylamine) is a low-molecularweight thiol compound that has been used as a radioprotective agent (16); as a treatment for acetaminophen intoxication (17); and as a therapy for the inherited metabolic disorder, cystinosis $(8,18,19)$. In addition, cysteamine has been shown to modify the properties of apo E-2, a mutant protein in which a cysteine substitution for arginine at amino acid residue 158 distinguishes it from the E-3 and E-4 isotypes $(7,8,20)$. The substitution is thought to be responsible for the impaired interaction of apo-E with cell surface receptors in type III hyperlipoproteinemia (the homozygous E-2 state). When the cysteine residues of apo E-2 are converted by cysteamine treatment to a positively charged lysine analogue, there is a marked increase in the binding activity (7). In addition, the apolipoprotein isoelectric pattern shifted after cysteamine treatment of cystinosis in a patient with homozygous apo E-2 (8).

The substitution of a cysteine for arginine-1689 in the Factor VIII light chain prevents thrombin cleavage at that site and thereby blocks FVIII-EH cofactor function (6). Because of its effect on apo E-2, we evaluated the ability of cysteamine to modify the mutant Factor VIII protein. We found that the addition of cysteamine at micro- to millimolar levels increased FVIII-EH VIII:C activity as much as 14-fold, as monitored by procoagulant (Figs. 1 and 2) and chromogenic (Fig. 3) assays. In addition, treatment of FVIII-EH with cysteamine restored light chain cleavage by thrombin (Fig. 4).

As the normal Factor VIII light chain has an odd number of cysteine residues, it is feasible that cysteine- 1689 forms a disulfide bond within the mutant protein. Although the locations of the nine normal Factor VIII light chain cysteine residues have been determined (residues 1,832, 1,858, 1,899, 1,903, 2,000,

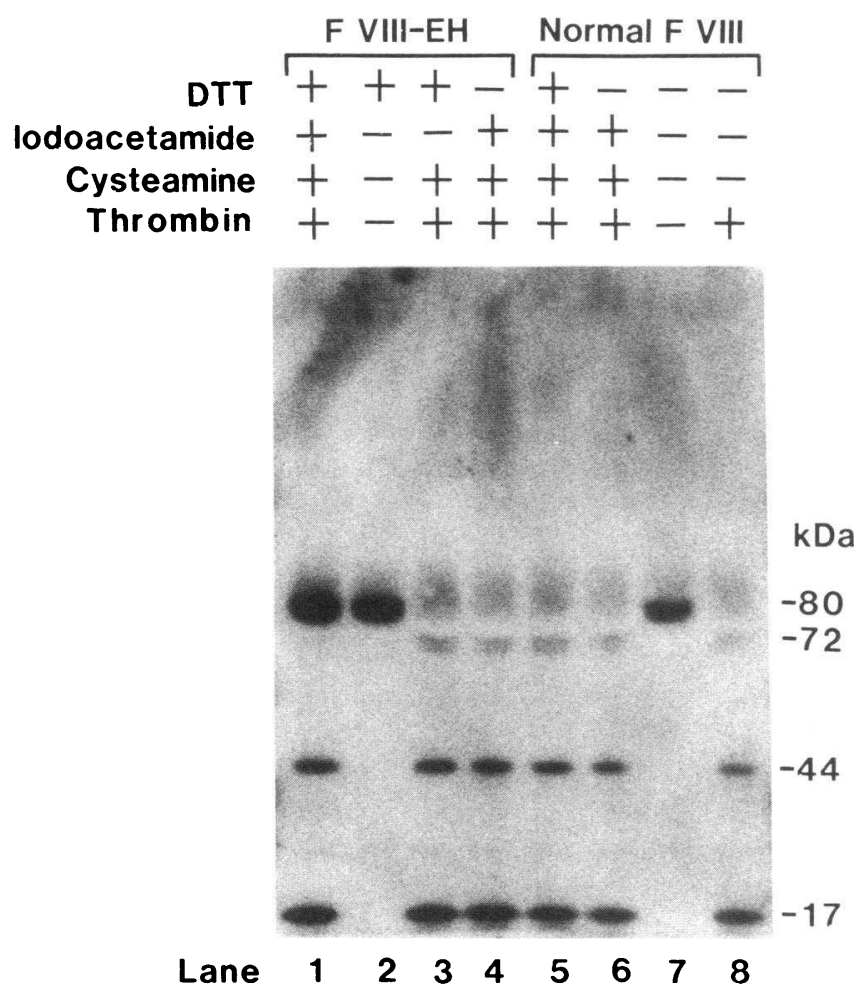

Figure 5. Effect of iodoacetamide on cysteamine-dependent FVIII-EH light chain cleavage. Initial incubation of the normal or FVIII-EH plasma (2 U VIII:Ag) with DTT $(5 \mathrm{mM})$ or BBS $\left(30 \mathrm{~min}\right.$ at $\left.37^{\circ} \mathrm{C}\right)$ was followed by addition of IA $(20 \mathrm{mM})$ or BBS for an additional 30 min. After dialysis, the treated plasmas were incubated overnight with anti-Factor VIII beads. Cysteamine (10 mM) or BBS was then added to the plasma/bead suspension for $30 \mathrm{~min}$ at $37^{\circ} \mathrm{C}$ before washing. All of the samples except those in lanes 2 and 7 were then incubated with $10 \mathrm{U}$ thrombin for $1 \mathrm{~h}$ at room temperature. The pattern for ARC-5 is shown here; similar data were obtained with ARC-10. 
$2,021,2,169,2,174$, and 2,326$)(21,22)$, their pairing in disulfide bonds is not known. There must be at least one free sulfydryl group, however, since there are no Factor VIII interchain disulfide bonds. As the FVIII-EH light chain has normal molecular mass on SDS-PAGE in the absence of added reducing agents (Fig. 4), we know that the mutant Factor VIII light chain does not form a new disulfide bond with some other plasma component. This is in contrast to the report of a mixed disulfide formed between antithrombin III-Northwick Park (arginine-393 to cysteine) molecules and albumin (23). Our data with thiol inhibitors (Table II and Fig. 5) suggest that the cysteine-1689 forms an intrachain disulfide in FVIII-EH.

Although we do not yet have direct evidence that cysteamine forms a covalent bond with cysteine-1689, the thrombin cleavage requirement for a positively charged amino acid at that residue (4) strongly suggests that a "pseudolysine" is formed when FVIII-EH is incubated with cysteamine. Pittman and Kaufman (4) showed by site-directed mutagenesis that the substitution of lysine for arginine at residue 1,689 does not reduce Factor VIII activity nor does it inhibit thrombin cleavage. On the other hand, substitution of isoleucine at that position resulted in a Factor VIII molecule that was resistant to thrombin cleavage and that had very little VIII:C ( 5\% of control) (4).

In addition to the cysteamine effect, reproducible FVIIIEH VIII:C augmentation was achieved using penicillamine, DTT, and dimethylaminoethanethiol (Table I). However, VIII:C enhancement by these compounds is due to a very different mechanism. They do not support FVIII-EH light chain cleavage by thrombin, and they appear to increase VIII:C by an effect on the Factor VIII-vWf interaction (15a, 24).

Data obtained with $S$-phosphocysteamine and the $S$ - $S$ cysteamine dimer (cystamine) indicate that a free sulfydryl is essential for the cysteamine interaction with FVIII-EH. However, other compounds with free sulfhydryl groups do not have an VIII:C augmenting effect when added to FVIII-EH (Table I). The negative data for cysteine are also instructive, for this compound is similar to cysteamine apart from the additional carboxyl group. Thus, compounds that can establish a thrombin cleavage site appear to need both a free sulfhydryl and a free amino group comparable to that present in lysine and arginine.

In vivo chemical modification of mutant FVIII-EH protein, if possible, would be beneficial for a small number of hemophiliacs. Although the frequency of the FVIII-EH mutation is not known with certainty, preliminary data suggest that it may be as high as 1 in every 170 hemophilic patients. It was detected in 2 of the first 17 CRM-positive plasmas that we evaluated, and CRM positivity is seen in $~ 5 \%$ of all patients with hemophilia A (13). To date, the FVIII-EH mutation has been detected by other laboratories in at least five other patients (25).

Although cysteamine has a wide variety of toxic effects when given at high doses (26), the administration of $60 \mathrm{mg} / \mathrm{kg}$ per $\mathrm{d}$ to patients with cystinosis appears to be well tolerated over long periods of time (8). This results in plasma concentrations between 50 and $100 \mu \mathrm{M}$. Indeed, regular oral administration at this dosage for as long as $6 \mathrm{yr}$ has been associated with only minor problems such as the foul taste and smell of cysteamine and occasional gastrointestinal upset (8). Since no serious adverse effects attributable to sulfhydryl group modification have been observed in 6 yr of regular cysteamine therapy at $60 \mathrm{mg} / \mathrm{kg}$ per $\mathrm{d}$, Gahl et al. (8) have suggested that even higher doses may be feasible in the treatment of life-threatening metabolic disorders.

It remains to be determined, however, whether a sufficiently high plasma cysteamine level can be obtained in vivo to significantly affect FVIII-EH VIII:C activity. The in vitro data are inconclusive, since reproducible increases in FVIII-EH VIII:C activity were obtained only at cysteamine concentrations $>100 \mu \mathrm{M}$ (Fig. 1). This is above the peak plasma cysteamine concentrations $(48.6 \pm 10.7$ [SD] $\mu \mathrm{M})$ obtained in the treatment of cystinosis (27). However, in patients with frequent recurrent hemarthroses, e.g., ARC-10 and the patient reported by O'Brien and Tuddenham (28) and Gitschier et al. (29), even a very small VIII:C increase might have a significant clinical benefit, and clinical trials will be necessary to evaluate the potential role of cysteamine in the management of FVIII-EH patients.

There are other coagulation factor deficiencies due to the substitution of cysteine for arginine, including Factor VIIIOkayama (30), Factor IX-Cardiff (also designated Factor IXAlbuquerque) (31, 32), prothrombin-Madrid (33), antithrombin III-Toyama (34), fibrinogens-Bergamo-I, Stonybrook-Kawaguchi and Osaka I (35-37), and fibrinogen-Tochigi (38). The likelihood of a benefit by addition of cysteamine will depend on the ability of that compound to interact with the mutant cysteine, as well as the impact of that interaction on that protein's function. Substitutions like that in FVIII-EH, the modification of a residue that is essential for thrombin cleavage, would seem to be the most likely to benefit from cysteamine addition. In each case, the effect of cysteamine can be evaluated in vitro, and, if positive, the possibility of treating the symptomatic disease could then be considered.

\section{Acknowledgments}

We thank Dr. Manoo Bhakta for providing ARC-10 plasma, Dr. Carol Fulcher for providing MAb J16D-9, Dr. John Fenton for providing purified human thrombin, Dr. William Gahl for bringing the potential use of cysteamine to our attention and for helpful discussions, Dr. Dorothea Scandella for helpful discussions and manuscript review, and Debbie Wilder for manuscript preparation.

These studies were supported by grants HL-36099, HL-44336, and RR-05737 from the National Institutes of Health.

\section{References}

1. Fulcher, C. A., J. R. Roberts, and T. S. Zimmerman. 1983. Thrombin proteolysis of purified factor VIII procoagulant protein: correlation of activation with generation of a specific polypeptide. Blood. 61:807-811.

2. Eaton, D., H. Rodriguez, and G. A. Vehar. 1986. Proteolytic processing of human factor VIII: correlation of specific cleavages by thrombin, factor $\mathrm{Xa}$, and activated protein $\mathrm{C}$ with activation and inactivation of factor VIII coagulant activity. Biochemistry. 25:505-512.

3. Kane, W. H., and E. W. Davie. 1988. Blood coagulation factors V and VIII: structural and functional similarities and their relationship to hemorrhagic and thrombotic disorders. Blood. 71:539-555.

4. Pittman, D. D., and R. J. Kaufman. 1988. Proteolytic requirements for thrombin activation of anti-hemophilic factor (factor VIII). Proc. Natl. Acad. Sci. USA. 85:2429-2433.

5. Hoyer, L. W., and R. T. Breckenridge. 1968. Immunologic studies of antihemophilic factor (AHF, factor VIII): cross-reacting material in a genetic variant of hemophilia A. Blood. 32:962-971.

6. Arai, M., M. Higuchi, S. E., Antonarakis, H. H. Kazazian, J. A. Phillips R. L. Janco, and L. W. Hoyer. 1990. Characterization of a thrombin cleavage site mutation (Arg 1689 to Cys) in the factor VIII gene of 2 unrelated patients with cross reacting material positive hemophilia A. Blood. 75:384-389.

7. Weisgraber, K. H., T. L. Innerarity, and R. W. Mahly. 1981. Abnormal 
lipoprotein receptor binding activity of human $\mathrm{E}$ apoprotein due to cysteine-arginine interchange at a single site. J. Biol. Chem. 257:2518-2521.

8. Gahl, W. A., R. E. Gregg, J. M. Hoeg, and E. Fisher. 1985. In vivo alteration of a mutant human protein using the free thiol cysteamine. Am. J. Med. Genet. 20:409-417.

9. Gahl, W. A., N. Bashan, F. Tietze, I. Bernardini, and J. D. Schulman. 1982. Cystine transport is defective in isolated leukocyte lysosomes from patients with cystinosis. Science (Wash. DC). 217:1263-1265.

10. Arai, M., H. Inaba, M. Higuchi, S. E. Antonarakis, H. H. Kazazian, M. Fujimaki, and L. W. Hoyer. 1989. Direct characterization of factor VIII in plasma: detection of a mutation altering a thrombin cleavage site (arginine-372 -histidine). Proc. Natl. Acad. Sci. USA. 86:4277-4281.

11. Breckenridge, R. T., and O. D. Ratnoff. 1962. Studies on the nature of the circulating anticoagulant directed against antihemophilic factor: with notes on an assay for antihemophilic factor. Blood. 20:137-149.

12. Seghatchian, M. J., and M. Miller-Anderson. 1978. A colorimetric evaluation of factor VIII:C potency. Med. Lab. Sci. 35:347-354.

13. Lazarchick, J., and L. W. Hoyer. 1978. Immunoradiometric measurement of the factor VIII procoagulant antigen. J. Clin. Invest. 62:1048-1052.

14. Hoyer, L. W. 1972. Immunologic studies of antihemophilic factor (AHF, factor VIII). IV. Radioimmunoassay of AHF antigen. J. Lab. Clin. Med. 80:822833.

15. Gawryl, M. S., and L. W. Hoyer. 1982. Immunologic studies of antihemophilic factor (AHF, VIII:C). VI. Characterization of antigenic determinants using human antibodies. Clin. Immunol. Immunopathol. 23:517-526.

15a. Aly, A. M., and L. W. Hoyer. 1992. Factor VIII-East Hartford (Arginine 1689 to Cysteine) has procoagulant activity when separated from von Willebrand factor. J. Clin. Invest. 89:1382-1387.

16. Purdie, J. W. 1979. A comparative study of the radioprotective effect of cysteamine, WR-2721, and WR-1065 in cultured human cells. Radiat. Res. 77:303-311.

17. Hughes, R. D., B. G. Gazzard, M. A. Hanid, P. N. Trewby, I. M. MurrayLyon, M. Davis, R. Williams, and J. R. Bennett. 1977. Controlled trial of cysteamine and dimercaprol after paracetamol overdose. Br. Med. J. 2:1395.

18. Thoene, J. G., R. G. Oshima, J. C. Crawhall, D. L. Olson, and J. A. Schneider. 1976. Intracellular cystine depletion by aminothiols in vitro and in vivo. J. Clin. Invest. 58:180-189.

19. Gahl, W. A., J. G. Thoene, J. A. Schneider, S. O'Regan, M. I. KaiserKupfer, and T. Kuwabara. 1988. Cystinosis: progress in a prototypic disease. Ann. Intern. Med. 109:557-569.

20. Fisher, E. A., and W. A. Gahl. 1982. Cysteamine in treatment of type III hyperlipoproteinemia. Lancet. i:1131-1132.

21. Wood, W. I., D. J. Capon, C. C. Simonsen, D. L. Eaton, J. Gitschier, B Keyt, P. H. Seeburg, D. H. Smith, P. Hollingshead, K. L. Wion, et al. 1984. Expression of active human factor VIII from recombinant DNA clones. Nature (Lond.). 312:330-337.

22. Toole, J. J., J. L. Knopf, J. M. Wozney, L. A. Sultzman, J. L. Buecker, D. D. Pittman, R. J. Kaufman, E. Brown, C. Shoemaker, E. C. Orr, et al. 1984. Molecular cloning of a cDNA encoding human antihaemophilic factor. Nature (Lond.). 312:342-347.

23. Erdjument, H., D. A. Lane, H. Ireland, M. Panico, V. Di Marzo, I. Blench, and H. R. Morris. 1987. Formation of a covalent disulfide-linked antithrombinalbumin complex by an antithrombin variant, antithrombin "Northwick Park." J. Biol. Chem. 262:13381-13384.
24. Aly, A. M., and L. W. Hoyer. 1991. Factor VIII-East Hartford (Arg 1689 Cys) activity is enhanced by chemicals that lead to thrombin cleavage or dissociation from von Willebrand factor. Thromb. Haemostasis. 65:942. (Abstr.)

25. Schwaab, R., M. Ludwig, L. Kochhan, J. Oldenburg, J. H. McVey, H. Egli, H. H. Brackmann, and K. Olek. 1991. Detection and characterization of two missense mutations at a cleavage site in the factor VIII light chain. Thromb. Res. 61:225-234.

26. Corden, B. J., J. D. Schulman, J. A. Schneider, and J. G. Thoene. 1981. Adverse reactions to oral cysteamine use in nephropathic cystinosis. Dev. Pharmacol. Ther. 3:25-30.

27. Smolin, L. A., K. F. Clark, J. G. Thoene, W. A. Gahl, and J. A. Schneider 1988. A comparison of the effectiveness of cysteamine and phosphocysteamine in elevating plasma cysteamine concentration and decreasing leukocyte free cystine in nephropathic cystinosis. Pediatr. Res. 23:616-620.

28. O'Brien, D. P., and E. G. D. Tuddenham. 1989. Purification and characterization of factor VIII 1,689-Cys: a nonfunctional cofactor occurring in a patient with severe hemophilia A. Blood. 73:2117-2122.

29. Gitschier, J., S. Kogan, B. Levinson, and E. G. D. Tuddenham. 1988. Mutations of factor VIII cleavage sites in hemophilia A. Blood. 72:1022-1028.

30. Shima, M., J. Ware, A. Yoshioka, H. Fukui, and C. A. Fulcher. 1989. An arginine to cysteine amino acid substitution at a critical thrombin cleavage site in a dysfunctional factor VIII molecule. Blood. 74:1612-1617.

31. Liddell, M. B., I. R. Peake, A. M. Taylor, D. P. Lillicrap, J. C. Giddings, and A. L. Bloom. 1989. Factor IX Cardiff: a variant factor IX protein that shows abnormal activation is caused by an arginine to cysteine substitution at position 145. Br. J. Haematol. 72:556-560.

32. Toomey, J., D. Stafford, and K. Smith. 1988. Factor IX Albuquerque (arginine 145 to cysteine) is cleaved slowly by factor XIa and has reduced coagulant activity. Blood. 72:312a. (Abstr.)

33. Diuguid, D. L., M.-J. Rabiet, B. C. Furie, and B. Furie. 1989. Molecular defects of factor IX Chicago-2 (Arg $145 \rightarrow$ His) and prothrombin Madrid (Arg $271 \rightarrow$ Cys): arginine mutations that preclude zymogen activation. Blood. 74:193-200.

34. Koide, T., S. Odani, K. Takahashi, T. Ono, and N. Sakuragawa. 1984 Antithrombin III Toyama: replacement of arginine- 47 by cysteine in hereditary abnormal antithrombin III that lacks heparin-binding ability. Proc. Natl. Acaa. Sci. USA. 81:289-293.

35. Reber, P., M. Furlan, E. A. Beck, G. Finazzi, M. Buelli, and T. Barbui. 1985. Fibrinogen Bergamo I (A- $\alpha 16 \mathrm{Arg} \rightarrow$ Cys): susceptibility towards thrombin following aminoethylation, methylation, or carboxyamidomethylation of cysteine residues. Thromb. Haemostasis. 54:390-393.

36. Miyata, T., S. Terukina, M. Matsuda, A. Kasamatsu, Y. Takeda, T. Murakami, and S. Iwanaga. 1987. Fibrinogens Kawaguchi and Osaka: an amino acid substitution of $\mathrm{A} \alpha$ arginine-16 to cysteine which forms an extra interchain disulfide bridge between the two A $\alpha$ chains. J. Biochem. Tokyo. 102:93-101.

37. Galanakis, D. K., A. Henschen, E. I. B. Peerschke, and M. Kehl. 1989. Fibrinogen Stony Brook, a heterozygous A $\alpha 16$ Arg $\rightarrow$ Cys dysfibrinogenemia Evaluation of diminished platelet aggregation support and of enhanced inhibition of fibrin assembly. J. Clin. Invest. 84:295-304.

38. Youshida, N., K. Ota, M. Moroi, and M. Matsuda. 1988. An apparently higher molecular weight gamma-chain variant in a new congenital abnormal fibrinogen Tochigi characterized by the replacement of gamma arginine- 275 by cysteine. Blood. 71:480-487. 